\title{
Räumliche Praktiken der Energiewende am Beispiel der Biogaserzeugung in Rheinland-Pfalz
}

\author{
Fabian Faller ${ }^{1}$
}

Eingegangen: 4. Mai 2015 / Angenommen: 20. April 2016 / Online publiziert: 23. Mai 2016

(C) Springer-Verlag Berlin Heidelberg 2016

Zusammenfassung Die Energiewende ist seit einigen Jahren Gegenstand intensiver akademischer Debatten. Ein Schwerpunkt liegt dabei auf regionalen Transformationen von konventionellen zu erneuerbaren Energiesystemen. Diese Regionen werden oft als Handlungsräume zwischen der lokalen und nationalen Ebene definiert und Transformationsprozesse werden in oder zwischen solchen Regionen analysiert. Gegenstand des vorliegenden Beitrags ist es, die Prozesse zu verstehen, die die Energieregionen hervorbringen. Wie werden sie konstituiert und transformiert? Welche geographischen Aspekte sind dabei bedeutsam? Eine qualitative Fallstudie über die Biogaserzeugung im westlichen Rheinland-Pfalz untersucht verschiedene Praktiken sowie Routinen der Energieerzeuger und beleuchtet die Entstehung, Stabilisierung und Veränderung von Energieregionen. Problemzentrierte Interviews mit 20 Anlagenbetreibern dienen als empirische Grundlage, um Aufschluss über individuelle Wahrnehmungen von Routinen und Praktiken zu gewinnen. Die Ergebnisse aus den Einzelfallstudien können zu übergeordneten Erkenntnissen generalisiert werden und zeigen, wie sich im Lauf der Zeit Praktiken, ihre räumliche Relevanz und ihre Bedeutung für die Energiewende verändern. Der Beitrag zeigt, dass sowohl individuelle (praktisches Wissen, kognitive Voraussetzungen) als auch strukturelle Kontexte (Zugang zu und Verfügbarkeit von Wissen, bestehende Netzwerke und politische Rahmenwerke) für die räumliche Bedeutung von Transformationspraktiken wichtig sind. So wird ersichtlich, dass über die Zeit Energieregionen verändert werden,

Dr. Fabian Faller

faller@geographie.uni-kiel.de

1 Institut für Geographie, Universität Kiel, Ludewig-Meyn-Str. 14, 24098 Kiel, Deutschland von eher subjektiven Räumen hin zu regionalen Netzwerkräumen mit ko-lokalisierten Akteuren und gemeinsamen Lerneffekten.

Schlüsselwörter Energiewende · Praktiken · Biogas · Regionalisieren · Praktikenkontexte

\section{Spatial Practices of the Energy Transition and the Case of Biogas Production in Rhineland-Palatinate}

Abstract The energy transition has received increasing scientific interest over the last decade. A main focus is set on regional transformations from conventional to renewable energies. There, regions are typically defined as action spaces nested between local and national policy levels, and transition processes are analyzed in or between such regions. Adding on this, the main purpose of the paper in hand is to understand the processes that lead to the formation and change of such regions. How get energy regions constituted and transformed? Which geographical aspects are important for these processes? A qualitative case study from western Rhineland-Palatinate in Germany focusing on biogas co-fermentation explores different practices and routines of energy producers that illuminate the constitution, stabilization and transformation of "energy regions". Problem-centered interviews are conducted with 20 plant operators to understand individual perceptions of routines and practices. The findings from these micro case studies are generalized and show the changing practices over time, their spatial connotations, and their importance for the energy transition. The paper discloses both, individual (practical knowledge and cognitive preconditions) and structural contexts (accessibility and availability of knowledge, existing networks and political frameworks) that are 
important for transition practices. Thereby, we show that over time the energy region changes from a subject oriented space to a regional network space bound to actors practicing in spatial proximity and building on mutual learning effects.

Keywords Energy transition - Social practices · Biogas · Regionalising $\cdot$ Practice contexts

\section{Einleitung}

Seit etwas über einem Jahrzehnt ist die Energiewende ein wichtiger Gegenstand der raumwissenschaftlichen Forschung. Dabei kommt regionalen Transformationen hin zu einer erneuerbaren Energieinfrastruktur vermehrt Aufmerksamkeit zu. Die Analyseeinheit „Region“"wird dabei verstanden als ein Handlungsraum, der in Übereinstimmung mit politisch-administrativen Raumeinheiten steht und zwischen der lokalen und nationalen Ebene liegt (vgl. Gailing/Röhring 2015: 37). Gesellschaftliche, energiebezogene Zusammenhänge werden innerhalb dieser Region oder zwischen verschiedenen Regionen betrachtet, Akteure und deren Netzwerke analysiert und wie sie diesen Handlungsraum prägen. Beispiele dafür sind die Analysen der Evolution von Akteuren und Unternehmensstrukturen der Windenergie in Deutschland (Kammer 2012), der räumlichen Diffusion der Fotovoltaikinstallationen in Deutschland (Rode/Weber 2011), der Wechselwirkungen von Politik und Markt bei der Entwicklung der Fotovoltaik in Deutschland (Dewald 2010), der regionalen Wertschöpfungseffekte unterschiedlicher Organisationsformen bei der Stromerzeugung aus Biomasse (Kosfeld 2012) oder der Entwicklungspfade der Offshore-Windenergieindustrie in Nordwestdeutschland (Mossig/Fornahl/Schröder 2010). Da die Analyseregion vorab gesetzt ist, wird deren Entstehung und Veränderung allerdings bisher nicht weiter hinterfragt (Hamhaber 2010: 16 f.). Ziel des vorliegenden Artikels ist es dagegen, das Verständnis über genau diese Entwicklung und Veränderung am Beispiel der Biogaserzeugung zu vertiefen und damit im Rahmen einer Fallstudie einen Beitrag zum weiteren Verständnis regionaler Transformationsprozesse im Kontext der Energiewende zu leisten.

Dazu rücken routinisierte Praktiken von Energieerzeugern in den Mittelpunkt, die beispielhaft Aufschluss über die Konstitution, Stabilisierung und Transformation einer „Energieregion“ liefern. Mit dem Fokus auf die Bioenergie lauten die forschungsleitenden Fragen: Wie konstituieren Biogaserzeuger im westlichen Rheinland-Pfalz im Wechselspiel mit anderen Akteuren einen Biogassektor und wie verändern sie diesen im Laufe der Zeit? Welche räumlichen Aspekte sind dafür von Bedeutung? Insbesondere die Entstehung und Verbreitung von Routinen werden als wesentlich für räumliche Transformationen wie die Energiewende zuerst konzeptionell herausgearbeitet und dann empirisch überprüft. Die explizite Einbeziehung des Aspekts ,Raum“ in Verbindung mit einem praktikenorientierten Akteursbegriff wird es darüber hinaus ermöglichen, die Evolution eines Bereichs der Energieerzeugung aus räumlicher Perspektive zu beleuchten. Regionen werden dabei als Kontexte für Praktiken begriffen. Als Fallstudie dient ein Untersuchungsgebiet im westlichen RheinlandPfalz. Dieses ist von einer Vielzahl installierter Biogasanlagen gekennzeichnet; bisher liegen aber keine raumwissenschaftlichen Forschungsarbeiten dazu vor. Der Zeitrahmen der qualitativen Untersuchung reicht von der Inbetriebnahme der ersten Anlage 1999 bis ins Jahr 2013, in dem der Großteil der empirischen Daten erhoben wurde. Dabei kann an Arbeiten aus anderen Disziplinen angeknüpft werden, die sich mit der Bioenergieerzeugung beschäftigen und Themen beleuchten wie technische Innovation (Decker/ Menrad/Berenz et al. 2007; Negro/Hekkert 2008; Wirth/ Markard/Truffer et al. 2013; Seiwald 2014), agrarisches Investitionsverhalten (Granoszewski/Reise/Spiller et al. 2009; Degenhart/Holstenkamp 2011; Granoszewski/Reise/Spiller et al. 2013), Finanzierung von Biogasanlagen (Schaper/ Beitzen-Heineke/Theuvsen 2008; Böttcher 2013), raumplanerische Steuerung (Kanning/Buhr/Steinkraus 2009; Kanning 2011; Wiehe/Rode/Kanning 2011) oder gesellschaftliche Akzeptanz (Roesch/Kaltschmitt 1999; Zoellner/ Schweizer-Ries/Wemheuer 2008; Griesen 2010).

Der Artikel gliedert sich wie folgt: Zuerst (Kapitel 2) werden als konzeptueller Ausgangspunkt die Transformationsforschung und die Praktikenforschung in ihren Kerngemeinsamkeiten beschrieben. Es werden drei Aspekte diskutiert. Erstens werden Transformationen als Resultat des Wandels von Praktiken dargestellt (Abschnitt 2.1). Zweitens wird aufgezeigt, dass dieser praktikentheoretische $\mathrm{Zu}$ gang ein Akteursbild über Subjekte erfordert, das sie als Träger von Routinen und Wissensbeständen ausgibt (Abschnitt 2.2). Und drittens wird die räumliche Dimension der Energiewende auf Transformationspraktiken zurückgeführt, wobei Regionen für soziale Praktiken als spezifische RaumZeit-Kontexte fungieren (Abschnitt 2.3). Daran schließen sich in Kapitel 3 einige methodische und analytische Überlegungen an, die auch das qualitative Untersuchungsdesign der empirischen Arbeit erläutern. Das vierte Kapitel wendet sich der empirischen Untersuchung zu und geht genauer auf Wissensbestände und Routinen der Biogaserzeugung sowie die entscheidenden Kontexte für Praktiken im Untersuchungsgebiet ein. Der Aufsatz schließt in Kapitel 5 mit einem Resümee, das die Kernerkenntnisse präsentiert und einen Ausblick auf weitere Forschungen zu Praktiken der Energiewende gibt. 


\section{Konzeptueller Ausgangspunkt: Transformationen als Praktiken}

In der internationalen raumwissenschaftlichen Literatur haben sich in der jüngeren Vergangenheit die Ansätze der Socio-Technical Transition Studies zur Analyse der Energiewende als langfristigem Prozess nicht nur bewährt, sondern auch als Forschungsheuristik etabliert (beispielsweise Essletzbichler 2012; Bridge/Bouzarovski/Bradshaw et al. 2013; Monstadt/Wolff 2015). Transformationen wie die Energiewende werden dort als soziotechnisch bezeichnet, da sie neben der Technikentwicklung auch Veränderungen von Märkten, Nutzerpraktiken, Politiken und Bedeutungszuschreibungen im Wechselspiel mit zuständigen Institutionen und der Umwelt beinhalten. Grundlegender Ausgangspunkt ist die Idee, dass sich technische Entwicklung und gesellschaftlicher Wandel gegenseitig beeinflussen und gemeinsam entwickeln (Ko-Evolution). Der Forschungsfokus liegt auf den Beziehungen zwischen diesen Aspekten. Ziel ist es, die gesellschaftlichen Voraussetzungen für die Entwicklung soziotechnischer Systeme und deren Einfluss auf die Gesellschaft zu verstehen und zu erklären. Gemäß einer Literaturanalyse von Markard/Raven/Truffer (2012) ist dabei die Energiewende der in der empirischen Forschung am stärksten repräsentierte Forschungsgegenstand.

Kritik richtet sich insbesondere an zwei Aspekte. Erstens ist nach wie vor unklar, worin sich die räumlich-regionale Dimension von Transformationen ausdrückt. Wie Markard/Raven/Truffer (2012: 962) beobachten, wird insbesondere in der empirischen Arbeit häufig von nationalstaatlichen oder subnationalen Räumen ausgegangen, ohne jedoch explizit zu fragen, ,why these boundaries were chosen and how they affect the findings and the generalizability of the results", also wie die Grenzen gewählt werden und ihre Einflüsse auf die Ergebnisse aussehen. Diesen Befund bestätigen auch Hamhaber (2010), Keppler (2013: 75 ff.) und Gailing/Röhring (2015) mit besonderem Augenmerk auf die Energiewendeforschung in Deutschland. Raum und Region werden als gegeben, als räumliche Ebene aufgefasst, aber die Frage der Konstitution derselben wird nicht tiefer gehend gestellt. Auch neuere Ansätze, die aus raumwissenschaftlicher Perspektive Akteursnetzwerke (Coenen/Benneworth/Truffer 2012) oder Entwicklungsdynamiken einzelner Techniken (Binz/Truffer/Coenen 2014; Seiwald 2014) fokussieren und dabei ein relationales Raumverständnis anlegen, um Aufschluss über räumliche Zusammenhänge zu gewinnen, konnten bisher dieses selbstgesetzte Ziel nicht erreichen. Dies liegt insbesondere an den auf Institutionen ausgerichteten Erklärungsansätzen. Denn so, wie Transformationen nicht einfach im Raum ablaufen, existieren auch Institutionen nicht per se in einem klar abgrenzbaren Raum. Institutionen werden durch Praktiken geschaffen, aufrechterhalten und transformiert (Feld-
man/Orlikowski 2011; Radwan/Kinder 2013), wodurch ihre Bedeutung für eine soziotechnische Transformation fernab von Raumeinheiten wie Regionen entsteht (Shove/Walker 2010; McMeekin/Southerton 2012). Es bietet sich also an, einen Ansatz zu wählen, der nicht Institutionen, sondern Praktiken der Transformation beleuchtet, um die räumlichen Zusammenhänge der Energiewende tiefer zu durchdringen.

Der zweite Kritikpunkt weist auf das unzureichende Verständnis in Bezug auf die Rolle von Akteuren hin. In den Socio-Technical Transition Studies wird, wie im Nachfolgenden kurz dargelegt, ein Akteursverständnis zugrunde gelegt, das auf den Aspekten begrenzter Rationalität, Routinen und Regeln beruht: Durch bewusste Entscheidungen tragen Akteure zur Erzeugung und Verbreitung von Techniken bei und das Zusammenwirken dieser Akteure ist entscheidend für Erfolg oder Misserfolg einer Transformation („Primat der agency“) (Bergek/Jacobsson/Carlsson et al. 2008; Binz/ Truffer/Coenen 2014). Beispielsweise seien Unternehmer kaum bereit, einen Betrieb aufzubauen, ohne eine staatliche ideelle oder materielle Unterstützung dafür erwarten zu können. Zugleich böten lokale oder nationale Regierungen kaum finanzielle Unterstützung an, ohne entsprechende Argumente dafür seitens der Unternehmer zu kennen, um ihre Politik zu rechtfertigen. Problematisch an diesem Akteursverständnis ist nach Shove und Walker (2007: 768 f.), dass Handlungsergebnisse und Effekte gezielter Interventionen nicht vorhersehbar und Systeme nicht unbedingt steuerbar seien, was aber zugleich Grundlage des angelegten Akteursverständnisses sei. In Übereinstimmung damit stellt Jørgensen (2012: 997) fest, dass Transformationsprozesse Ergebnisse reproduktiver und rekonfigurierender Aktionen sind. In diesen Aktionen würden bestehende Konfigurationen und Institutionen reflektiert werden, ,but the stability of these institutions may be interpreted very differently by actors" (Jørgensen 2012: 997). Folglich ergeben sich individuell unterschiedliche Interpretationen institutioneller Rahmenbedingungen. Daher scheint es sinnvoll, Akteure aus dieser individuellen Perspektive heraus zu verstehen. In Verbindung mit dem ersten Kritikpunkt wird deutlich, dass es zielführend ist, die Praktiken individueller Akteure in ihrem Kontext zu beleuchten (Späth/Rohracher 2010; Jørgensen 2012), um erstens die Entstehung und Verbreitung routinisierter Praktiken zu begreifen und damit zweitens räumliche Transformationsprozesse tiefer gehend zu verstehen.

\subsection{Transformation als Wandel von Praktiken}

Im Kontext der Energiewende sind Praktiken interessant, die auf Energieerzeugung und -verbrauch abzielen. Im vorliegenden Beitrag wird der von Jones und Murphy (2010) für die wirtschaftsgeographische Forschung vorgeschlagene Praxisbegriff zugrunde gelegt, definiert als „the stabilized, 
routinized, or improvised social actions that constitute and reproduce economic space, and through and within which diverse actors (eg, entrepreneurs, workers, caregivers, consumers, firms) and communities (eg, industries, places, markets, cultural groups) organize materials, produce, consume, and/or derive meaning from the economic world" (Jones/Murphy 2010: 367). Die explizite Bezugnahme auf die Raumkonstitution sowie das Zusammenspiel von Akteuren und Gemeinschaften unterstreicht den Mehrwert eines praktikentheoretischen Ansatzes für das Interesse des vorliegenden Beitrags. Wie Reckwitz (Reckwitz 2002; Reckwitz 2003) in seiner umfassenden Diskussion der Praktikentheorien darlegt, sind Praktiken mehr als einzelne Handlungen. Sie erfordern Kollektivität und Koexistenz von Menschen sowie erkennbare und wiederholbare Muster zusammenhängender Einzelhandlungen. Beispiele für Praktiken sind Investitionsentscheidungen sowie der Bau und der alltägliche Betrieb einer Energieerzeugungsanlage, die Gründung und Unterhaltung eines Interessenverbandes oder Konsumentscheidungen. Die Gesamtheit dieser Praktiken erzeugt beispielsweise Facetten der sozialen Dimension der Biogaserzeugung. Und die Summe aller Praktiken konstituiert aus praxistheoretischer Sicht die soziale Welt. Diese soziale Welt ist einerseits durch Routinisiertheit gekennzeichnet, also alltäglich wiederkehrenden Handlungsweisen, sowie andererseits durch die „Unberechenbarkeit interpretativer Unbestimmtheiten" (Reckwitz 2003: 294), also dem ebenso alltäglichen Misslingen und Neuinterpretieren von Handlungsweisen bzw. sozialen Praktiken.

Um die Transformation bestimmter Praktiken besser zu verstehen und damit Aufschluss über Wendeprozesse zu gewinnen, wird in der Literatur insbesondere der Analyse der Entstehung und Verbreitung von Routinen ein Mehrwert zugesprochen. Unter Routinen können aus praxistheoretischer Sicht „repetitive, recognizable patterns of interdependent actions, carried out by multiple actors" (Feldman/ Pentland 2003: 95) verstanden werden. Wesentlich für ihre Entstehung und die Verbreitung dieser wiederholten und erkennbaren Muster sind Wissensbestände sowie Interaktionen. Wissen ist im praxistheoretischen Verständnis immer praktisches Wissen (knowing in practice). Es äußert sich in konkreten Handlungen, die Artefakte, Individuen und Netzwerke miteinander in Beziehung setzen (Faulconbridge 2006). Das in den Praktiken vereinte Wissen wird zugleich zur Basis für die wiederholte Ausübung einer Routine (Orlikowski 2002). Die Verbreitung einer Routine erfolgt durch den Austausch dieser Wissensbestände und damit durch interaktive Praktiken. Sie kann verstanden werden ,as situated practices dispersed in time and space" (Radwan/Kinder 2013: 2454). Durch Austausch können sich auch einzelne Elemente der Praxis ändern und damit auch die Handlungen einzelner Akteure (um)definiert werden (Sydow/Lerch/
Staber 2010: 176). Damit wird die translokale Mobilisierung von Routinen zu einem komplexen Prozess, der in engem Zusammenhang mit institutionellen Kontexten, sozialen Strukturen und individuellen kognitiven Voraussetzungen steht. Der jeweilige Kontext eröffnet Handlungsräume, auf die sich Akteure in ihren Praktiken berufen können. Daher ist es nach Radwan und Kinder (2013: 2446) auch nicht vordergründig relevant, wie beispielsweise nationalstaatliche Gesetzgebung konstituiert wird, sondern vielmehr, wie sie als Bezugsrahmen für Routinen wirkt und deren Diffusionsprozesse beeinflusst. Interpretationen und Wahrnehmungen individueller Akteure, die Mobilisierung von Wissensbeständen in Praktiken und deren Austausch sowie die damit verbundene Herausbildung von Routinen sind demnach zentrale Mechanismen von Transformationen.

\subsection{Akteure der Transformationen}

Akteure sind der Ansatzpunkt für die Praktikenforschung. Denn es sind Akteure, ,who 'carry" and 'carry out' social practices. [...] As carriers of a practice, they are neither autonomous nor the judgmental dopes who conform to norms: They understand the world and themselves, and use know-how and motivational knowledge, according to the particular practice“ (Reckwitz 2002: 256). Als individuelle Akteure - oder Subjekte - bilden sie „den Kreuzungspunkt unterschiedlicher Verhaltens/Wissenskomplexe sozialer Praktiken“ (Reckwitz 2003: 296). Als Träger von Routinen sind es die Akteure, die durch intersubjektiven Austausch die Verbreitung der Routinen durchführen. Einerseits können damit, ausgehend von den Praktiken mehrerer Subjekte, Muster dieser sozialen Praktiken herausgearbeitet werden, die routinisiertes Handeln offenlegen. Andererseits werden durch den empirischen Zugriff auf Subjekte heterogene Wissensbestände und Formen ihres Austauschs sichtbar. So können Transformationen als Praxisphänomen begriffen werden: Praktiken ändern sich im Lauf der Zeit, womit Transformationen ablaufen, dass heißt, geänderte Praktiken führen zu Transformationen (vgl. Shove/Walker 2007: 768).

\subsection{Raum als Ergebnis transformativer Praktiken}

Für das Verständnis der räumlichen Dimension von Transformationen wird ein konstruktivistischer Raumbegriff zugrunde gelegt. Raum dient dabei der Beschreibung räumlicher Praktiken. Er kann nach Werlen ,als ein Element des Handelns verstanden werden, das [...] in unterschiedlicher Ausformung ein Mittel [der Praxis] darstellt" (Werlen 2009: 154). Es steht also nicht ein wie auch immer verfasster Raum im Zentrum, sondern die sozialen Praktiken seiner Hervorbringung; Räume sind Teilaspekte sozialer Praktiken. Diese Praktiken bilden das Beziehungsverhältnis von 
physisch-materiellen Komponenten (oder Artefakten), subjektiven Sinnzuschreibungen und -deutungen (als Grundlage für individuelles Wissen) und gesellschaftlichen Strukturen (Normen, Werte und Routinen, auch in Netzwerken) als ,Raum‘ ab. So kann Raum auch nicht als Erklärung für soziale Strukturen herangezogen werden. Denn räumliche Strukturen sind eine „Ausdrucksform über Handeln vermittelter sozialer Strukturen. [...] Doch gerade weil sie ,Ausdruck' sind, können sie mit diesen nicht gleichgesetzt werden“ (Werlen 2007: 119).

Dennoch ist Raum für soziale Praxis von Bedeutung, allerdings nicht per se, sondern als Kontext, beispielsweise in Form von „Regionen“. Regionen werden hier als Kontexte für Praktiken begriffen. Diese Kontexte umfassen nach Werlen (2007: 180), der sich dazu auf Giddens beruft, normative Rechte, aber auch Verpflichtungen und mögliche Sanktionen. Region bezeichnet demnach einen spezifischen RaumZeit-Kontext, der für Praktiken einen Rahmen setzt, zugleich aber auch durch routinisierte Praktiken entsteht. Oder in anderen Worten: Eine Region repräsentiert einen symbolisch markierten beziehungsweise bedeutungsvollen Ausschnitt des Lebenskontextes, der physisch-materiell rückgebunden werden kann. Zugleich sind Regionen als ,sinnhaft konstituiert“ aufzufassen (Werlen 2007: 191). Sie sind ,sozialer Art [und] nicht naturhaft vorgegeben, sondern Ausdruck von Konstitutionsleistungen und der sozialen Reproduktionsprozesse im Rahmen alltäglicher Praxis“ (Werlen 2007: 191). Mit alltäglichen Praktiken meint Werlen das routinisierte und unhinterfragte Handeln von Menschen.

Aus diesem Raumverständnis kann gefolgert werden, dass sich die Praktiken der Raumkonstitution im zeitlichen Verlauf ändern und damit den Raum der Transformationen - wie die Energieregion - immer wieder konstituieren, stabilisieren oder transformieren. Aus praxistheoretischer Perspektive interessiert also nicht die (Energie-)Region an sich, sondern das Regionalisieren, also deren Produktion und Transformation.

\section{Methodisches und analytisches Vorgehen}

Vor dem Hintergrund des skizzierten Akteursverständnisses bildet der empirische Individualismus nach Boudon/ Bourricaud (1992: 223-226) die Grundlage zur Analyse räumlicher Transformationsprozesse sowie der Entstehung und Verbreitung damit verbundener routinisierter Praktiken. Im empirischen Individualismus rücken Routinen, ihre Ursprünge und Effekte sowie ihre Stabilisierung und ihr Wandel in den Mittelpunkt, indem konsequent die subjektiven Erfahrungsbereiche, Wissensbestände und deren Ursachen und Folgen für Routinen fokussiert werden. So werden Wandel und Verstetigung sozialer Praktiken zum analytischen Hauptgegenstand. Durch die Fokussierung auf Sub- jekte, im vorliegenden Fall die Betreiber von Biogasanlagen in ihrem spezifischen Kontext im westlichen Rheinland-Pfalz, können Erkenntnisse über deren Praktiken gewonnen werden, welche Routinen sich im Laufe der Zeit herausbilden beziehungsweise ändern, wie sich also Routinen in bestimmten räumlichen Kontexten transformieren. Der methodische Zugang dafür ist qualitativer Art, um Aufschluss über Wissensbestände der Betreiber zu erhalten, die Rückschlüsse auf die Praktiken und deren Transformation zulassen.

Das empirische Material besteht aus 20 leitfadengestützten, problemzentrierten Interviews, die mit Betreibern von Biogasanlagen im westlichen Rheinland-Pfalz geführt wurden (Mikrostudien; im Text nummeriert von M-01 bis M-20), um über ihre Praktiken und Routinen Aufschluss zu erhalten. Durch Auswertung der Betreiberdatenbank des Netzbetreibers amprion (2014) konnte eine umfassende Datenbank mit über 85 Biogasanlagen im Untersuchungsgebiet erstellt werden, die durch das Telefonbuch mit Kontaktdaten erweitert wurde. Aus der erstellten Betreiberdatenbank wurde jeweils zufällig ein Betreiber angerufen. Wenn er den Anruf beantwortete, konnte mit ihm in der Regel direkt ein Interviewtermin vereinbart werden. Der Leitfaden umfasste Fragen zu folgenden Themen, die sich aus einer umfassenden Literaturrecherche ergaben (Faller 2016: 51-125): Geschichte des Engagements im Biogassektor und Gründe dafür; Bedeutung der Anlage in der alltäglichen Arbeit; technische Aspekte der Anlage; Entwicklung der Anlage; Rolle von Partnern und sozialen Netzwerken, die für die Biogaserzeugung als wichtig wahrgenommen wurden sowie deren Entwicklung; Einschätzungen der Gesamt- sowie der politischen Situation der Biogaserzeugung; Wahrnehmung gesellschaftlicher Entwicklungen im Bereich erneuerbare Energien. Die Interviews wurden standardorthographisch transkribiert und thematisch codiert. Es wurden individuelle Schilderungen und Wahrnehmungen der Interviewten fokussiert, wie sie von ihrer Tätigkeit im Biogasbereich berichten und damit verschiedene Elemente der vorgebrachten Routinen und Praktiken aufzeigen. Diese Elemente und deren sich verändernde Bedeutung wurden entlang einer Zeitachse abgetragen. Somit konnten die synchrone sowie die diachrone Entwicklung der mit der Biogasanlage in Beziehung gebrachten Artefakte, Individuen und Netzwerke abgebildet werden.

Die Analyse des empirischen Materials beruht auf der qualitativen Inhaltsanalyse. Dieses von Mayring (2000) entwickelte Verfahren dient der systematischen Aufbereitung des empirischen Materials hinsichtlich Inhalt und Qualität. Es ist ein häufig verwendetes und gut dokumentiertes Vorgehen, weshalb hier auf eine genauere Vorstellung verzichtet wird. Es ist möglich, die verschiedenen individuellen Interpretationen und Wahrnehmungen routinisierter Praktiken zu extrahieren und zu vergleichen, wie deren Austausch 
Abb. 1 Das Untersuchungsgebiet im westlichen RheinlandPfalz. (amprion 2014)

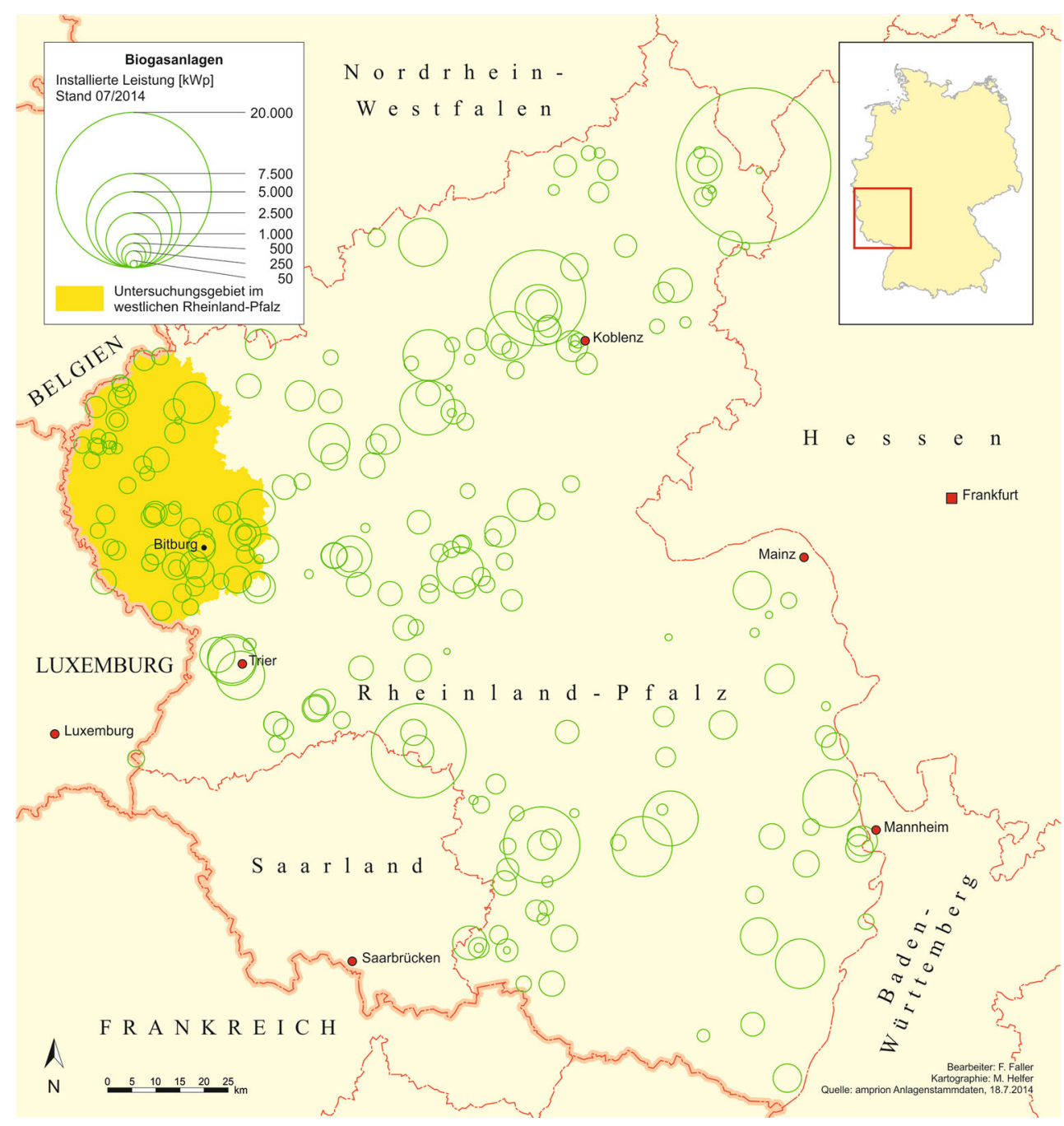

geschildert wird. So können die Prozesse der Entstehung und Verbreitung von Routinen im Biogassektor im Untersuchungsgebiet herausgearbeitet werden. Neben individuellen Faktoren und Einblicken in die spezifische Situation vor Ort kann demnach auch ein Verständnis über die regionalen und nationalen Kontexte gewonnen werden. Damit werden die Zusammenhänge der individuellen Konstitutionsleistungen der Betreiber und der damit verbundenen Transformationsprozesse im Raum erklärbar.

\section{Praktiken der Biogaserzeugung im Transformationsprozess im westlichen Rheinland-Pfalz}

Wie konstituieren Biogaserzeuger im westlichen Rheinland-Pfalz im Wechselspiel mit anderen Akteuren einen Biogassektor und wie verändert sich dieser im Laufe der Zeit? Welche räumlichen Aspekte sind dafür von Bedeutung? Wie diskutiert, liegt der Schlüssel zur Beantwortung der Forschungsfragen in der Entstehung und Verbreitung von Routinen der Biogaserzeugung im Untersuchungsgebiet (s. Abb. 1). Wissensbestände bilden dabei die Grundlage für den Umgang mit der Biogasanlage und damit für die Praktiken der Transformation an sich. Somit rücken die Mobilisierung und der Austausch von Wissensbeständen, die sich in der Entstehung und Verbreitung von Routinen ausdrücken, in den Forschungsmittelpunkt, und damit Interpretationen und Wahrnehmungen der Anlagenbetreiber, die Einblicke in die räumlichen Kontexte liefern.

Abb. 2 zeigt die Entwicklung des Anlagenbestands im Untersuchungsgebiet. Mit der vermehrten Nutzung der Biogastechnik wurden auch die relevanten Wissensbestände und Routinen räumlich verbreitet. Die befragten Betreiber im westlichen Rheinland-Pfalz gingen in den Interviews auf diverse biogasspezifische routinisierte Praktiken ein: das Auswählen von Ressourcen, deren Beschaffung, das Instandhalten der Anlage, das alltägliche Ausführen anfallender kleinerer Arbeiten, die Lektüre von Fachliteratur und das Besuchen von Fachausstellungen und Messen sowie das 


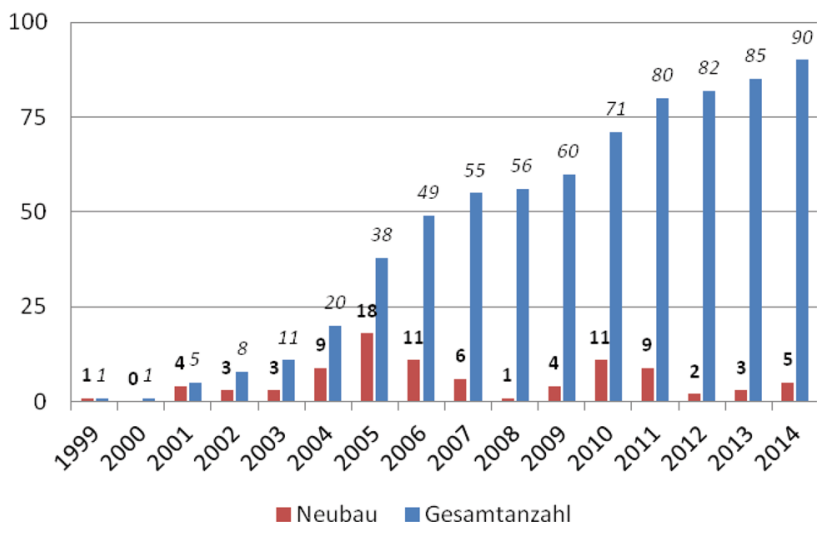

Abb. 2 Bestand und Anzahl neu errichteter Biogasanlagen im Untersuchungsgebiet (1999-2014). (amprion 2014)

Zusammenarbeiten mit Dienstleistern. Im Folgenden werden diese Aspekte genauer beleuchtet und aufgearbeitet, welche „Praktikenkontexte“ für deren Entstehung und Verbreitung relevant waren, wie sich also die Energieregion herausbildete.

\subsection{Wissen und Routinen im ,,regionalen“ Biogassektor}

Als 1999 die erste Anlage im Untersuchungsgebiet errichtet wurde, gab es dort kaum Routinen der und Wissen über Biogaserzeugung. So äußerte der Betreiber, dem die erste Biogasanlage gehört: „Es gab nicht einmal einen vernünftigen Motorenhersteller, da musste man ja sogar danach suchen“ (M-06). Zum damaligen Zeitpunkt waren in Deutschland bereits 850 Anlagen in Betrieb (Statista 2015) und es existierten auf Biogasanlagen spezialisierte Motorenhersteller (Schnell seit 1992 und Dreyer \& Bosse seit 1997). Dass der befragte Betreiber anscheinend nicht davon wusste, deutet darauf hin, dass die Wissensbestände nicht ubiquitär verfügbar waren. Für fehlende Routinen ist der Ressourceneinsatz ein Beispiel: Derselbe Betreiber hatte wenige Monate nach Betriebsstart große Probleme mit der Anlage. Die Kernursache dafür war, dass der eingesetzte Hühnertrockenkot sandhaltig war und sich der Sand im Fermenter ablagerte, Leitungen verstopfte und die Anlage durch mechanischen Abrieb beschädigte. „Woher hätte man das wissen sollen? Hatte ja keiner die Erfahrung gemacht bis dato" (M-06). Auch hier zeigen sich mangelnde individuelle Wissensbestände, denn einige der 850 Anlagen liefen unter Einsatz von Hühnertrockenkot. Es stellt sich also die Frage, wie in der Folge Wissensbestände und Routinen der Biogaserzeugung entwickelt und verbreitet wurden, um eine Energieregion zu konstituieren und zu transformieren.

\subsubsection{Wissensquellen, -aneignung und-verbreitung im westlichen Rheinland-Pfalz.}

Die Interviewten gingen auf diverse Wissensquellen, Formen der Wissensaneignung und -verbreitung für den Betrieb ihrer Anlagen ein, die für die Entwicklung von Routinen wesentlich waren. Die Lektüre von Fachzeitschriften sowie der gezielte Besuch von Fachausstellungen und Messen wurden als wichtig angeführt, um neues Wissen zu gewinnen. Dabei wurde von vielen Befragten im westlichen Rheinland-Pfalz das „Dienstleistungszentrum Ländlicher Raum" (DLR) mit Sitz in Bitburg als Plattform benannt, das bis heute Informationsmaterial erarbeitet, eigene Veranstaltungen organisiert und externe Tagungen bewirbt. Austausch und Zusammenarbeit mit erfahrenen einzelnen Experten wurden ebenfalls mit großer Bedeutung belegt. Etablierte Anlagenhersteller und Planungsbüros stellten für nahezu alle Betreiber wichtige Quellen für Wissensbestände dar und lieferten Einblicke in ihre Routinen. Ein Befragter (M-15) schilderte, dass sich durch die Einbindung in ein Projekt, das auch heute noch verschiedene Energieträger verbindet, vor Ort Lernvorteile ergaben. Da das Projekt seiner Auskunft nach eine gewisse Anziehungskraft besaß, seien zahlreiche Besuchergruppen auf seinen Hof gekommen: Politiker waren interessiert, informierten sich über Biogaserzeugung und wurden damit für den Betreiber zugänglich; Gruppen von Studierenden und interessierte Forscherteams begleiteten die Projektentwicklung und gaben Rückmeldungen, von denen der Betreiber profitieren konnte. Zudem lieferte das mitverantwortliche Energieversorgungsunternehmen Expertisen für den Betrieb und das Management der Anlage. Ein anderer Betreiber (M-04) ging auf die Stabilisierung von Routinen durch Interaktion mit dem Anlagenhersteller ein. Er verfolgte den Bau der Anlage durch diesen sehr genau, ließ sich alle mechanischen Elemente und Arbeitsschritte im Detail erklären und war somit auf spätere Reparaturarbeiten vorbereitet: Praktiken wurden so weitergegeben.

Das gemeinsame Erschließen von Wissensbeständen unter Betreibern ist eine zweite Art der Ausbreitung von Routinen. Gerade in der Anfangsphase waren Studienreisen hilfreich, um Wissen über biologische Prozesse und technische Varianten auszutauschen. Zudem war es insbesondere in der Anfangsphase für diverse Betreiber hilfreich, direkt von Kollegen zu lernen. Als beispielsweise ein Interviewter Probleme mit der Biogasqualität hatte, wandte er sich an einen erfahrenen Kollegen, der auf praktisches Wissen und damit auf Routinen verwies und zu dem Betreiber sagte: „Mach mal den gelben Hahn da auf und halt das Feuerzeug da hin. Wenn die Flamme richtig blau ist, dann ist das Gas gut" (M-11). Der gleiche Betreiber führte an, dass sich diese Situation inzwischen geändert habe. Heute hätten die Betreiber die Möglichkeit, sich mit einem der vielen 
anderen Kollegen im Umfeld auszutauschen. Dieser Austausch unter Betreibern war für nahezu alle Befragten ein wichtiger Aspekt. Auf der Basis von Bekanntschaft und Vertrauen können sie Routinen weitergeben und damit zur Routinisierung bestimmter Praktiken beitragen.

Eine dritte Art der Stabilisierung oder Transformation von Routinen ist das Einbinden individuellen Vorwissens der Betreiber in Praktiken der Biogaserzeugung. Einige Interviewpartner waren bereits im Energiebereich tätig, betrieben Fotovoltaik- oder Windenergieanlagen. Damit hatten sie erste Erfahrungen im Energiesektor sammeln können, wussten beispielsweise über technische Anforderungen an Transformatoren und damit verbundene Regulierungen Bescheid oder kannten verschiedene Förderungsmöglichkeiten. Auch Tätigkeiten in Ausschüssen und Gremien der Landwirtschaft, wie beispielsweise im Bauernverband, in der Landjugend oder im Meisterprüfungsausschuss wurden benannt, durch die die Betreiber regelmäßig Einblicke in laufende Verfahren oder politische Entwicklungen erhielten. So war beispielsweise ein Betreiber in der Zeit vor der ersten Biogasanlage als Beratungslandwirt für die Deutsche Saatveredelung aktiv und konnte so Netzwerke in den Handel aufbauen, von denen er noch heute profitiert: „Die kommen mit Neuerungen ab und zu auf mich zu und dann wird immer probiert, die interessanten Sachen. Der Bezug ist bestehen geblieben und dadurch kommt man vielleicht mal schneller an Sachen dran wie andere" (M-17). Diese verschiedenen Praktikenkontexte ermöglichen den Transfer spezifischer individueller Wissensbestände aus anderen Bereichen in Alltagsabläufe der Biogaserzeugung, womit sich dort bestehende Routinen schrittweise ändern.

Zudem spielt Learning by doing eine wesentliche Rolle. Ein Betreiber äußerte, nach dieser Form gefragt: „Learning by doing, das ist auf jeden Fall das Wesentliche, muss ich ganz klar sagen, wo ich mir jetzt mein Wissen quasi angeeignet habe" (M-04). Diese Aussage ist typisch für die Betreiber im Untersuchungsgebiet. Technische Steuerung, mechanische Wartung und Fütterung der Anlage sind stark von praktischem Erfahrungswissen abhängig. Das eigene Handanlegen ist dabei wichtig: „Wenn du das selbst machst und selbst planst und dir selbst deine Lieferanten 'raussuchst und dann bist du im Lernprozess schon von dem Tag an, wo du das Erste machst" (M-08). Dabei können Learning by doing und Learning by interacting auch nahe beieinander liegen, wie ein Befragter äußerte: „Wenn ein Problem kam, musste man sich damit befassen und ja, daraus hat man dann gelernt. Der Kontakt untereinander war dann schon da oder man wusste: der hat den gleichen Motor, den kannst du mal fragen“" (M-12). So können durch räumliche Nähe gemeinsame Wissensbestände aufgebaut und verbreitet werden, was die Voraussetzung für die Verbreitung von Routinen ist.

\subsubsection{Veränderung von Routinen der Biogaserzeugung}

Die gewonnenen Einblicke in verschiedene Routinen der Biogaserzeugung bilden die Grundlage für die nachfolgende Analyse des Transformationsprozesses im Untersuchungsgebiet. Denn die Stabilisierung, Konstitution und Transformation der Routinen spiegelt zugleich - wie im konzeptionellen Teil dargelegt - die Herausbildung der Energieregion wider.

Auswählen und Beschaffen von Ressourcen. Die ausreichende Versorgung mit Ressourcen war für die Betreiber zu jeder Zeit wichtig. Dabei orientierte sich die Auswahl wesentlich an den gesetzlich möglichen oder begünstigten Substraten in Form von Bonuszahlungen für Nachwachsende Rohstoffe (eingeführt mit der EEG-Novelle 2004) oder den Einsatz von Gülle (Güllebonus, EEG 2009). Im Jahr 2003 hatten immerhin vier der elf Betreiber neben nachwachsenden Rohstoffen und Gülle auch organische Abfälle (,Biomüll“) mit in Verwendung. 2004 stellten alle vollständig auf nachwachsende Rohstoffe und Gülle um. Damit ergab sich auch, dass die Marktpreise für Agrarprodukte größere Bedeutung erhielten. Dass in den Jahren 2004 und 2005 fast ein Drittel aller Anlagen im Untersuchungsgebiet neu ans Netz ging (s. Abb. 2), kann auch auf diesen Aspekt zurückgeführt werden. Denn zwischen 2001 und 2005 reduzierte sich der durchschnittliche Verkaufspreis von Weizen in Deutschland, von dem auch der Maispreis wesentlich abhängt, zweimal beträchtlich - 2001 auf 2002 von 113 auf 104 Euro pro Tonne und von 2004 auf 2005 von 118 auf 93 Euro pro Tonne (Statista 2015). Für viele Betreiber bildete das ein sehr relevantes Einstiegskriterium in die Biogaserzeugung, um Einkommensausfälle - insbesondere aus dem Verkauf von Weizen - zu kompensieren, aber auch, da die wesentlichen Ressourcen für die Biogaserzeugung auf einmal günstiger waren. In Verbindung mit den Einspeiseregularien ergab sich wenig später eine hohe Nachfrage nach vor allem Mais, was auch zu Preissteigerungen beitrug (Februar 2008: 186 EUR/t). Seitdem schwankt der Marktpreis kontinuierlich und erheblich. Diverse Betreiber äußerten, dass sie gelernt hätten, mit dieser Situation umzugehen, dass sie sich in gewisser Weise an knappe Ressourcenbestände und hohe Preise gewöhnt hätten, sodass keine grundlegenden Änderungen für Auswahl oder Beschaffen von Ressourcen nach dem Jahr 2005 benannt wurden.

Instandhalten der Anlage. Kleinere Reparaturarbeiten führten die meisten Betreiber seit jeher eigenständig durch. Oftmals hatten sie selbst oder ein Familienmitglied technisches Interesse oder eine entsprechende Ausbildung, sodass diese Tätigkeiten in Eigenarbeit durchgeführt werden konnten. Die Instandhaltung der Anlage nehme, so alle Befragten, phasenweise zwar einen großen Teil der 
Tab. 1 Entwicklung der Anzahl von Ausstellern und Besuchern der Fachmesse „Biogas“ von 2009 bis 2015, nach Fachverband Biogas (2015: 2) und Faller (2016: 230)

\begin{tabular}{llll}
\hline Jahr & Aussteller & Besucher & Ausstellungsfläche in qm \\
\hline $\mathbf{2 0 0 9}$ & 240 & 3182 & 4116 \\
$\mathbf{2 0 1 0}$ & 285 & 3635 & 10.000 \\
$\mathbf{2 0 1 1}$ & 342 & 6911 & 11.400 \\
$\mathbf{2 0 1 2}$ & 431 & 7043 & 15.100 \\
$\mathbf{2 0 1 3}$ & 446 & 8065 & 17.200 \\
$\mathbf{2 0 1 4}$ & 406 & 7554 & 17.500 \\
$\mathbf{2 0 1 5}$ & 348 & 6283 & 13.500 \\
\hline
\end{tabular}

Arbeit in Anspruch, der einwandfreie Betrieb sei aber aus finanziellen Gründen entscheidend. Durch die zunehmende Technisierung der Anlagen wurden ab Mitte der 2000erJahre zusätzliche Instandhaltungsarbeiten an der Elektronik und aufgrund zunehmenden Alters der Motoren umfassende Wartungsarbeiten nötig. Beides ließen alle Befragten von externen Dienstleistern durchführen. Dies begründeten sie vor allem mit individuellem Wissensmangel sowie mit dem Anspruch auf Gewährleistung und dem Versicherungsschutz. Da Wartungsarbeiten zudem zu jeder Zeit anfallen könnten, sei die möglichst permanente Verfügbarkeit von Dienstleistern wichtig. Ein Betreiber äußerte dazu: „Das haben sie eigentlich bei jeder Anlage. Die sind auf regionale Strukturen angewiesen, weil Dienstleister einfach vor Ort sind und 24 Stunden Bereitschaft haben und das bringt natürlich sehr große Vorteile" (M-02). Die Zusammenarbeit findet im Untersuchungsgebiet folglich vorrangig mit bekannten und ansässigen Unternehmen statt. Die Instandhaltung trägt so zur Herausbildung der Energieregion bei, da Subjekte in regionalen Netzwerken ihre Routine stabilisieren.

\section{Alltägliches Ausführen anfallender kleinerer Arbeiten.} Die alltäglichen Praktiken, die unmittelbar an der Anlage durchzuführen sind, schilderte ein Betreiber anschaulich: „Wenn das richtig eingespielt ist, ist das im Prinzip nur einmal Rundlatschen und Gucken und grad die Fütterungsmengen, die kW-Zahlen, Gaszahlen aufschreiben. Das ist ja alles in ein paar Minuten gemacht und ich habe ja auch in ein paar Minuten gesehen, ob alles richtig läuft" (M-05). Zudem äußerten die meisten Befragten, dass der Umgang mit der Biogasanlage ähnlich sei wie der bereits bekannte und routinisierte Umgang mit Kühen. Transformiert haben sich die Alltagspraktiken allerdings im Zuge der Technisierung der Betriebe. Seit wenigen Jahren werden Störungsmeldungen der Biogasanlage, aber auch der Melk- und Fütterungsroboter, per SMS oder App auf die Mobiltelefone der Betreiber gesendet (z. B. Meldung einer durch Steine blockierten Fütterungsschnecke, eines Ausfalls der Pumpe oder einer leckgeschlagenen Leitung). Somit ist es nicht mehr unbedingt nötig, mehrmals täglich die Anlage zu be- gehen. Allerdings entstehen dadurch neue Alltagskonstellationen: „Manchmal verfluche ich die Abhängigkeit vom Handy!“ (M-19). Durch zunehmende Erfahrung mit den Meldungen können die Befragten heute zwischen akuten und weniger akuten Meldungen unterscheiden. Diese Befunde deuten darauf hin, dass sich der Umgang mit den meist mechanischen Problemen an der Anlage routinisiert hat. Zum einen ist dieser auf die Übertragung stabiler Praktiken aus anderen Bereichen zurückzuführen und zum anderen auf Transformationen biogasspezifischer Routinen.

Lektüre der Fachpresse und Besuche von Fachausstellungen und Messen. Der Besuch von Messen sowie die Lektüre von Fachliteratur wurden für die Betreiber im Lauf der Zeit wichtiger. Während um die Jahrtausendwende erste Berichte in der Fachpresse über das Thema Biogaserzeugung erschienen, spielten Messen noch keine Rolle. Ab der zweiten Hälfte der 2000er-Jahre wurde auch auf Branchentreffen wie der „Eurotier“ (einer Leitmesse für Tierhaltung) oder der „Agritechnika“ (einer Landtechnik-Ausstellung) zunehmend über Energieerzeugung und dabei insbesondere Biogas berichtet. Ein Vergleich der letzten Jahre des größten Branchentreffens „Biogas“ zeigt, wie sich die Fachmesse seit 2009 vergrößerte (s. Tab. 1). 2014 und 2015 nahmen weniger Aussteller und Besucher teil; da die Jahre außerhalb des betrachteten Zeitraums liegen, können darüber keine vertiefenden Informationen aus dem empirischen Material gewonnen werden. Auch die landwirtschaftliche Presse griff das Thema Biogaserzeugung nach Aussagen der Befragten in den letzten Jahren immer stärker auf. Beispielsweise wurden diverse Sonderausgaben zu dem Thema aufgelegt. Damit ergaben sich auch neue Formen der Wissensaneignung: Messefahrten und Lektüre sind heute für alle Befragten Routine.

Zusammenarbeiten mit Dienstleistern. Beratung durch Planungsbüros, Anlagenhersteller oder andere Experten thematisierten alle Interviewpartner. Bis einschließlich 2001 hatten die Betreiber im Untersuchungsgebiet drei Alternativen für die Planung der Anlage: Sie konnten Berater von außerhalb des Untersuchungsgebietes beauftragen, Ex- 


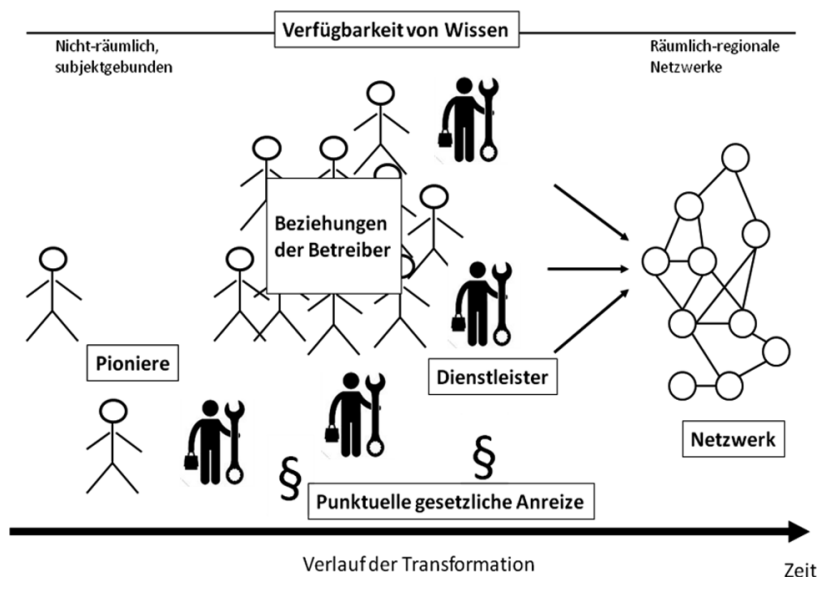

Abb. 3 Kontexte für Praktiken der Biogaserzeugung im Lauf der Zeit

perten aus sonstigen Praxisfeldern zu Rate ziehen oder die Planung und Kalkulation weitgehend eigenständig durchführen. Bereits 2002 gab es im gesamten Untersuchungsgebiet verschiedene auf Biogas spezialisierte Unternehmen, Techniklieferanten, Planungsbüros, Bauunternehmen oder Firmen, die alles aus einer Hand lieferten (Wirtschaftlichkeitsberechnung, Genehmigungsphase, Ausschreibungen). Routinen des Biogassektors hatten sich herausgebildet und stabilisiert. Seit Ende der 2000er-Jahre haben die privaten Dienstleister eine sehr große Bedeutung für die Betreiber. So äußerten einige, dass sie aus verschiedenen Gründen auf Planungsbüros angewiesen waren: „Also wenn man das auch sieht, was für einen Umfang das hat und was für Vorkenntnisse man da haben muss, also da ist man auch komplett überfordert. Deswegen müssen da erfahrene Leute dran“ (M-05). Zudem wurde erwähnt, dass insbesondere die im Untersuchungsgebiet tätigen Büros mit den einzelnen Sachbearbeitern bei den Behörden bekannt seien, was für den Ablauf der Genehmigungsverfahren sehr positiv sei. Für die vergangenen Jahre legten alle Befragten dar, dass die Bedeutung von Dienstleistern von fundamentaler Bedeutung sei und insbesondere im Untersuchungsgebiet tätige Büros bevorzugt würden.

Wie zu erkennen ist, ist der Wandel von Wissensbeständen und Routinen gleichermaßen Ausdruck sich ändernder Kontexte wie eine mögliche Ursache dieser Kontextänderungen. Im Folgenden wird daher genauer auf diese Kontexte eingegangen.

\subsection{Praktikenkontexte für das westliche Rheinland- Pfalz}

Wie deutlich wurde, gab es diverse Kontexte, die zur Stabilisierung, Konstitution und Transformation von Praktiken der Biogaserzeugung beitrugen. Diese waren zum einen subjektspezifisch (individuelles Erfahrungswissen, kognitive Voraussetzungen, praktisches Wissen) und zum ande- ren struktureller Art (Verfügbarkeit von Wissensbeständen, Netzwerke des Biogassektors, nationalstaatliche Rahmenwerke). Diese Differenzierung ermöglicht es, genauere Einblicke in Praktikenkontexte und damit die Energieregion zu gewinnen.

Subjektspezifische Kontexte variierten von Betreiber zu Betreiber. Erstens gehörte das individuelle Erfahrungswissen als begünstigender Faktor im Umgang mit neuen Praktiken dazu. Energiebezogenes Vorwissen, beispielsweise Erfahrungen mit Genehmigungsprozeduren oder Kenntnisse diverser Förderungsmöglichkeiten, fällt in diesen Bereich. Zweitens, aber eng damit verbunden, waren die landwirtschaftlichen Kontexte der Befragten. Dabei entfalteten individuelle Interpretationsmuster und kognitive Voraussetzungen Wirkung (Jones/Murphy 2010). So kann beispielsweise die betriebliche Diversifizierung durch den Aufbau einer Biogasanlage als Reaktion auf schwankende Marktpreise interpretiert werden, für die die spezifische betriebliche Situation eines Jeden ausschlaggebend ist. Der dritte subjektspezifische Kontext spiegelt sich im Learning by doing wider. Die eigene Anlage als Artefakt wurde zum Kontext der Routinenbildung, zum Gegenstand der alltäglichen praktischen Auseinandersetzung, die Know-how erforderte und damit den Aufbau praktischen Wissens begünstigte (Reckwitz 2002).

Im Lauf der Zeit waren verschiedene strukturelle Kontexte für alle Betreiber von Bedeutung (s. Abb. 3). In der Untersuchung wurde dabei erstens die Wichtigkeit der strukturellen Verfügbarkeit von Wissen deutlich. Zeitschriften, Messen, Berater, staatliche oder private Dienstleister stellten für alle Betreiber wichtige Kontexte ihrer Praktiken dar, die für die Aneignung und Verbreitung von Wissen eine große Rolle spielten. Damit in Zusammenhang standen zweitens Netzwerke des Biogassektors. Durch deren Entwicklung wurden Routinen herausgebildet und verbreitet. Während zu Beginn insbesondere Einzelkontakte zu Pionieren und mit benachbarten Betreibern wichtig waren, wurden im Lauf der Zeit strukturelle Beziehungen unter Betreibern, aber auch zwischen Betreibern und privaten (Wartung, Planung etc.) wie auch staatlichen Dienstleistern (z.B. das „Dienstleistungszentrum Ländlicher Raum“ ) immer bedeutsamer. Damit verschob sich auch die räumliche Dimension der Praktikenkontexte von teilweise nichträumlichem, subjektgebundenem Wissen hin zu räumlichregionalen Netzwerken. Drittens waren nationalstaatliche Rahmenwerke von struktureller Wichtigkeit. Vor allem das Erneuerbare-Energien-Gesetz (EEG) mit seiner Vergütungsstruktur wurde von allen Betreibern praktisch genutzt. Investitionsanreize, Einflüsse auf die Ressourcenwahl und steigende administrativen Anforderungen waren Aspekte, die von den Betreibern vorgebracht wurden und die auf diese staatlichen Praktikenkontexte zurückzuführen sind. 
Die Umsetzung dieser Kontexte in Praktiken führte zu einer, Verräumlichung' der Kontexte. Sie entfalteten Wirkung im Handeln der Subjekte. Die Kontexte wurden durch routinisierte Praktiken reproduziert. Die entstehenden Routinen wurden innerhalb von Netzwerken ausgetauscht und verändert. Die Praktikenkontexte bildeten zugleich die Energieregion ab. So, wie sich Kontexte im Laufe der Zeit änderten, änderte sich also auch die Energieregion. So kann gefolgert werden: Deuten Subjekte Kontexte neu oder entfalten andere Kontexte zusätzlich Wirkung, verändert sich die Energieregion. Daher sind die Praktiken gleichermaßen Ausdruck und Bedingung der Transformation.

\section{Resümee}

Der vorliegende Beitrag befasste sich damit, wie Biogaserzeuger im westlichen Rheinland-Pfalz im Wechselspiel mit anderen Akteuren einen Biogassektor konstituieren, wie sie diesen im Laufe der Zeit verändern und welche räumlichen Aspekte dafür von Bedeutung sind. Ziel war es, das Verständnis über die Entwicklung und Veränderung der Biogaserzeugung und ihrer räumlichen Zusammenhänge zu vertiefen und damit zum weiteren Verständnis regionaler Transformationsprozesse der Energiewende beizutragen. Es wurde deutlich, dass ein analytischer Fokus auf Praktiken das Potenzial bietet, kontextspezifische Forschung durchzuführen. Somit konnten für das Untersuchungsgebiet die aus Sicht der Betreiber von Biogasanlagen entscheidenden Kontexte herausgearbeitet werden.

Es zeigte sich, dass die Entstehung und Verbreitung von Wissen und Routinen von entscheidender Bedeutung für das Verständnis regionaler Transformationsprozesse ist. Insbesondere die Praktikenforschung bietet dafür konzeptionelle Werkzeuge (s. Kapitel 2). Subjekte werden als Träger der Praxis verstanden. Die Analyse der Praktiken innerhalb eines Untersuchungssamples kann dann auf Unterschiede der Praktiken im zeitlichen Verlauf hinweisen. Diese Änderungen lassen zugleich Rückschlüsse auf die entscheidenden Praktikenkontexte zu, sodass es insgesamt möglich wird, regionenbildende Transformationsprozesse zu beschreiben. Denn erst durch die praktische Auseinandersetzung mit konkreten Techniken, wie einer Biogasanlage, entfalten die Rahmenbedingungen und spezifischen Kontexte der Energiewende für die Subjekte Wirkung. Dies geschieht in bestimmten räumlichen Zusammenhängen, den Praktikenkontexten. Sie bilden zugleich die Energieregionen der Biogaserzeugung. Energieregionen analytisch als Räume der Praxis aufzufassen und nicht als Raumeinheiten der Analyse ermöglicht es, die praktische Bedeutung der Kontexte für regionale Transformationsprozesse zu erfassen.

Aus dem qualitativen Vorgehen der Fallstudie ergab sich die Möglichkeit, die als wesentlich erachteten Praktiken der Transformation des Biogassektors im westlichen Rheinland-Pfalz herauszuarbeiten. Denn trotz individueller Interpretationen und unterschiedlicher Perspektiven der Befragten konnten gemeinsame Kontexte für Praktiken identifiziert werden, wie sie auch von Späth/Rohracher (2010) für Leitbilder der Energiewende identifiziert wurden. Somit wurden etablierte Praktiken und Routinen der Biogaserzeugung herausgearbeitet und deren Verbreitung untersucht. Dabei konnte aufgezeigt werden, dass im Lauf der Zeit Bedeutungsverschiebungen zwischen übergeordneten und fallstudienspezifischen Kontexten auftreten. Es wurde deutlich, dass (a) unterschiedliche Formen der Wissensverbreitung zwischen Subjekten oder innerhalb von Netzwerken, (b) die Entstehung routinisierter Praktiken durch das Verknüpfen individueller Erfahrungen mit denen anderer Subjekte oder (c) die Bezugnahme auf nationalstaatliche Regelungen entscheidende Mechanismen der Bildung und Verbreitung von Praktiken im Biogassektor sind. Dabei spielten Kontakte zwischen Betreibern von Biogasanlagen, aber auch die Interaktion mit Beratern, Dienstleistern oder Verwaltungsmitarbeitern eine wichtige Rolle. Insgesamt entfalteten sowohl subjektspezifische als auch strukturelle Kontexte für Praktiken Bedeutung. Individuelles Wissen, kognitive Voraussetzungen und praktisches Wissen auf der einen und die Verfügbarkeit von Wissen, von Netzwerken sowie nationalstaatliche Rahmenwerke auf der anderen Seite prägten die untersuchte Energieregion in der Form eines Transitionsoder Praktikenraums.

So lässt sich schlussfolgern, dass durch die Verknüpfung verschiedener Praktiken Energieregionen erzeugt und stabilisiert beziehungsweise verändert werden. Der Praktikenraum unterscheidet sich von dem in der Energiewendeforschung zugrunde gelegten Handlungsraum insbesondere darin, dass er eben nicht auf ,bestehende regionale Raumkonstrukte (z. B. politisch-administrativer Art)“ (Gailing/Röhring 2015: 40) Bezug nimmt, sondern die raumzeitliche Transformation räumlicher Bezugnahmen in routinisierten Praktiken hervorhebt.

Weitere Studien könnten sich davon ausgehend mit der Bedeutung alternativer Praktiken beschäftigen. Was geschieht, wenn einzelne Betreiber von Routinen abweichen? Werden sie von bestimmten Praktikenkontexten zurück zur Routine gebracht? Verbreiten sich die alternativen Praktiken und werden damit einzelne Routinen geändert? Kann dies sogar zum Wandel ganzer Kontexte beitragen? Und wie entfalten andere Kontexte wie „Landwirtschaftsregionen" Wirkung? Um die komplexen räumlichen Prozesse der Energiewende besser zu verstehen, scheint es vor dem Hintergrund der vorliegenden Ergebnisse sinnvoll, stärker auf die performative Dimension der verschiedenen Subjekte und Netzwerke zu fokussieren. Wie dargelegt, folgt ein Großteil der bisherigen Studien dazu institutionellen Ansätzen. Diese können, wie gezeigt, durch eine 
praktikentheoretische Herangehensweise ergänzt werden. Aus wissenschaftstheoretischer Sicht könnte ein nächster Schritt sein, die Wechselbeziehungen der je gewonnenen Erkenntnisse im Kontext der Energiewende intensiver zu beleuchten.

Für die räumliche Politik und Planung der Energiewende lassen sich aus den vorgestellten Ergebnissen tiefere Einblicke in die Praktiken der Energieerzeugung gewinnen. Diese könnten in politische und planerische Prozesse einfließen. Ein Verständnis von Energieregionen, wie es hier vorgeschlagen wird, könnte zudem den Vorteil bieten, den komplexen Beziehungen des Energiesektors besser Rechnung zu tragen und zugleich spezifische Kontexte zu berücksichtigen. Anstatt Energieregionen an administrativen Grenzen festzumachen oder sie durch funktionale Zusammenhänge abzugrenzen, könnten sie als gegenstandspezifische Kontexte aufgegriffen werden, die räumlich flexibel die jeweils bedeutsamen Elemente umfassen. Dies würde auch für die räumliche Politik und Planung der Energiewende die Möglichkeit eröffnen, produktiv mit den kontextspezifischen Potenzialen und Hemmnissen umzugehen, die sich aus der jeweiligen Gemengelage von Elementen und Kontexten der Praktiken ergeben.

Danksagung Ich danke den Mitgliedern des ARL-Arbeitskreises „Energie“ für die produktive Zusammenarbeit. Die präsentierten empirischen Ergebnisse entstammen meiner Tätigkeit am Institut für Geographie und Raumplanung an der Universität Luxemburg.

\section{Literatur}

amprion (2014): Anlagenstammdaten (Stand: 31.12.2014). http:// www.amprion.net/eeg-anlagenstammdaten-aktuell (15.07.2015).

Bergek, A.; Jacobsson, S.; Carlsson, B.; Lindmark, S.; Rickn, A. (2008): Analyzing the functional dynamics of technological innovation systems: A scheme of analysis. In: Research Policy 37, 3, 407-429.

Binz, C.; Truffer, B.; Coenen, L. (2014): Why space matters in technological innovation systems - Mapping global knowledge dynamics of membrane bioreactor technology. In: Research Policy 43, $1,138-155$.

Böttcher, J. (2013): Projektfinanzierung eines Biogas-Projektes. In: Böttcher, J. (Hrsg.): Management von Biogas-Projekten. Rechtliche, technische und wirtschaftliche Aspekte. Berlin, Heidelberg, $17-49$.

Boudon, R.; Bourricaud, F. (1992): Soziologische Stichworte. Ein Handbuch. Opladen.

Bridge, G.; Bouzarovski, S.; Bradshaw, M.; Eyre, N. (2013): Geographies of energy transition: Space, place and the low-carbon economy. In: Energy Policy 53, 331-340.

Coenen, L.; Benneworth, P.; Truffer, B. (2012): Towards a spatial perspective on sustainability transitions. In: Research Policy 41, 6, 968-979.

Decker, T.; Menrad, K.; Berenz, S.; Wagner, R. (2007): Regulation and innovation in biogas technology in selected European countries. In: International Journal of Public Policy 2, 1/2, 89-108.

Degenhart, H.; Holstenkamp, L. (2011): Finanzierungspraxis von Biogasanlagen in der Landwirtschaft. Eine empirische Untersuchung zu Stand und Entwicklungslinien. Wiesbaden.
Dewald, U. (2010): Wechselwirkungen von Politik und Markt: Entwicklung der Photovoltaik in Deutschland. In: Schüssler, F. (Hrsg.): Geographische Energieforschung. Strukturen und Prozesse im Spannungsfeld zwischen Ökonomie, Ökologie und sozialer Sicherheit. Tagungsband zur Jahrestagung 2009 des Arbeitskreises Geographische Energieforschung. Frankfurt am Main, 83-105.

Essletzbichler, J. (2012): Renewable Energy Technology and Path Creation: A Multi-scalar Approach to Energy Transition in the UK. In: European Planning Studies 20, 5, 791-816.

Fachverband Biogas (2015): 24. Internationale Jahrestagung und Fachmesse. Veranstaltungsanalyse. Nürnberg. https://www.biogastagung.org/Filestore.aspx/ Veranstaltungsanalyse_BIOGAS_2015_D.pdf?fair=biogas\& type $=$ file $\&$ key $=36232 \mathrm{a} 19-\mathrm{ebcf}-46 \mathrm{~b} 7-\mathrm{a} 0 \mathrm{c} 1-\mathrm{cdc} 5 \mathrm{f} 2 \mathrm{~d} 36 \mathrm{acd} \&$ language $=$ de $\&$ filegroup $=\&$ filetype $=$ file $\&$ indexfile $=$ true (08.04.2016).

Faller, F. (2016): Energetisches Regionalisieren. Transformationspraktiken der Energiewende am Beispiel der Biogaserzeugung. Frankfurt am Main. = Études luxembourgeoises/Luxemburg-Studien, 10.

Faulconbridge, J. (2006): Stretching tacit knowledge beyond a local fix? Global spaces of learning in advertising professional service firms. In: Journal of Economic Geography 6, 4, 514-540.

Feldman, M.; Orlikowski, W. (2011): Theorizing Practice and Practicing Theory. In: Organization Science 22, 5, 1240-253.

Feldman, M.; Pentland, B. (2003): Reconceptualizing organizational routines as a source of flexibility and change. In: Administrative Science Quarterly 48, 13, 94-118.

Gailing, L.; Röhring, A. (2015): Was ist dezentral an der Energiewende? Infrastrukturen erneuerbarer Energien als Herausforderungen und Chancen für ländliche Räume. In: Raumforschung und Raumordnung 73, 1, 31-43.

Granoszewski, K.; Reise, C.; Spiller, A.; Mußhoff, O. (2009): Entscheidungsverhalten landwirtschaftlicher Betriebsleiter bei Bioenergie-Investitionen. Erste Ergebnisse einer empirischen Untersuchung. Göttingen. = Diskussionspapier des Departments für Agrarökonomie und Rurale Entwicklung der Universität Göttingen/DARE Discussion Papers, 0911.

Granoszewski, K.; Reise, C.; Spiller, A.; Mußhoff, O. (2013): Growth of Biogas Production in German Agriculture: An Analysis of Farmers' Investment Behaviour. In: Ruppert, H.; Kappas, M.; Ibendorf, J. (Hrsg.): Sustainable Bioenergy Production - An Integrated Approach. Dordrecht, Heidelberg, 241-292.

Griesen, M. (2010): Akzeptanz von Biogasanlagen. Aachen (zugleich Dissertation an der Rheinischen Friedrich-Wilhelms-Universität Bonn). = Bonner Studien zur Wirtschaftssoziologie, 34 .

Hamhaber, J. (2010): Humangeographische Zugänge in der Geographischen Energieforschung vom euklidischen Raum zu sozial konstruierten Raumbezügen. In: Schüssler, F. (Hrsg.): Geographische Energieforschung. Strukturen und Prozesse im Spannungsfeld zwischen Ökonomie, Ökologie und sozialer Sicherheit. Tagungsband zur Jahrestagung 2009 des Arbeitskreises Geographische Energieforschung. Frankfurt am Main, 9-19. = Schriften zur internationalen Entwicklungs- und Umweltforschung, 27.

Jones, A.; Murphy, J. (2010): Theorizing practice in economic geography: Foundations, challenges, and possibilities. In: Progress in Human Geography 35, 3, 366-392.

Jørgensen, U. (2012): Mapping and navigating transitions - The multilevel perspective compared with arenas of development. In: Research Policy 41, 6, 996-1010.

Kammer, J. (2012): Die Windenergieindustrie. Evolution von Akteuren und Unternehmensstrukturen in einer Wachstumsindustrie mit räumlicher Perspektive: Mitteilungen der Geographischen Gesellschaft in Hamburg. Stuttgart.

Kanning, H. (2011): Energetische Biomassenutzung im ländlichen Raum - Naturräumliche Auswirkungen des Biogaspfades und 
planerische Perspektiven für ein regionales (Energie-)Ressourcenmanagement. In: Tietz, H-P.; Hühner, T. (Hrsg.): Zukunftsfähige Infrastruktur und Raumentwicklung. Handlungserfordernisse für Ver- und Entsorgungssysteme. Hannover, 191-217. = Forschungs- und Sitzungsberichte der Akademie für Raumforschung und Landesplanung, 235.

Kanning, H.; Buhr, N.; Steinkraus, K. (2009): Erneuerbare Energien - Räumliche Dimensionen, neue Akteurslandschaften und planerische (Mit)Gestaltungspotenziale am Beispiel des Biogaspfades. In: Raumforschung und Raumordnung 67, 2, 142-156.

Keppler, D. (2013): Handlungsmöglichkeiten regionaler Akteure beim Ausbau erneuerbarer Energien. Grenzen regionalwissenschaftlich fundierter Empfehlungen und Erweiterungsmöglichkeiten durch techniksoziologische Konzepte. (Dissertation an der Technischen Universität Berlin). Berlin.

Kosfeld, R. (2012): Regionale Wertschöpfungseffekte unterschiedlicher Organisationsformen bei der Stromerzeugung aus Biomasse. In: Informationen zur Raumentwicklung 9/10, 555-561.

Markard, J.; Raven, R.; Truffer, B. (2012): Sustainability transitions: An emerging field of research and its prospects. In: Research Policy 41, 6, 955-967.

Mayring, P. (2000): Qualitative Inhaltsanalyse. In: Forum: Qualitative Sozialforschung 1, 2, Art. 22.

McMeekin, A.; Southerton, D. (2012): Sustainability transitions and final consumption: practices and socio-technical systems. In: Technology Analysis \& Strategic Management 24, 4, 345-361.

Monstadt, J.; Wolff, A. (2015): Energy transition or incremental change? Green policy agendas and the adaptability of the urban energy regime in Los Angeles. In: Energy Policy 78, 213-224.

Mossig, I.; Fornahl, D.; Schröder, H. (2010): Heureka oder Phoenix aus der Asche? Der Entwicklungspfad der Offshore-Windenergieindustrie in Nordwestdeutschland. In: Zeitschrift für Wirtschaftsgeographie 54, 3-4, 222-223.

Negro, S.; Hekkert, M. (2008): Explaining the success of emerging technologies by innovation system functioning: the case of biomass digestion in Germany. In: Technology Analysis \& Strategic Management 20, 4, 465-482.

Orlikowski, W. (2002): Knowing in Practice: Enacting a Collective Capability in Distributed Organizing. In: Organization Science 13, 4, 249-273.

Radwan, L.; Kinder, S. (2013): Practising the diffusion of organizational routines. In: Environment and Planning A 45, 10, 2442-2458.

Reckwitz, A. (2002): Toward a Theory of Social Practices. A Development in Culturalist Theorizing. In: European Journal of Social Theory $5,2,243-263$.

Reckwitz, A. (2003): Grundelemente einer Theorie sozialer Praktiken: Eine sozialtheoretische Perspektive. In: Zeitschrift für Soziologie $32,4,282-301$.
Rode, J.; Weber, A. (2011): Die räumliche Diffusion von Photovoltaikinstallationen in Deutschland. In: Helbich, M.; Deierling, H.; Zipf, A. (Hrsg.): Theorie und quantitative Methoden in der Geographie. Kolloquiumsbeiträge. Heidelberg, 57-69. = Heidelberger Geographische Bausteine, 19.

Roesch, C.; Kaltschmitt, M. (1999): Energy from biomass - do nontechnical barriers prevent an increased use? In: Biomass and Bioenergy 16, 5, 347-356.

Schaper, C.; Beitzen-Heineke, C.; Theuvsen, L. (2008): Finanzierung und Organisation landwirtschaftlicher Biogasanlagen: Eine empirische Untersuchung. In: Yearbook of Socioeconomics in Agriculture 1, 1, 39-74.

Seiwald, M. (2014): The (up)scaling of renewable energy technologies: experiences from the Austrian biomass district heating niche. In: Moravian Geographical Reports 22, 2, 44-54.

Shove, E.; Walker, G. (2007): CAUTION! Transitions ahead: politics, practices, and sustainable transition management. In: Environment and Planning A 39, 4, 763-770.

Shove, E.; Walker, G. (2010): Governing transitions in the sustainability of everyday life. In: Research Policy 39, 4, 471-476.

Späth, P.; Rohracher, H. (2010): 'Energy regions': The transformative power of regional discourses on socio-technical futures. Research Policy 39, 4, 449-458.

Statista (2015): Verkaufspreis von Weizen in Deutschland in den Jahren 2001 bis 2014 (in Euro pro 100 Kilogramm). http://de.statista.com/statistik/daten/studie/182308/umfrage/ verkaufspreise-fuer-weizen-in-deutschland/ (18.02.2016)

Sydow, J.; Lerch, F.; Staber, U. (2010): Planning for path dependence? The case of a network in the Berlin-Brandenburg optics cluster. In: Economic Geography 86, 2, 173-195.

Werlen, B. (2007): Sozialgeographie alltäglicher Regionalisierungen. Band 2: Globalisierung, Region und Regionalisierung. Stuttgart. $=$ Erdkundliches Wissen, 119 .

Werlen, B. (2009): Geographie/Sozialgeographie. In: Günzel, S. (Hrsg.): Raumwissenschaften. Frankfurt am Main, 142-158.

Wiehe, J.; Rode, M.; Kanning, H. (2011): Auswirkungen der Biogasproduktion auf Natur und Landschaft. Biomasseanbau, Naturschutz und Steuerung. In: Zeitschrift Ökologisches Wirtschaften 26, 3, 22-25. = Schwerpunktheft „Erneuerbare Energie Regionen“.

Wirth, S.; Markard, J.; Truffer, B.; Rohracher, H. (2013): Informal institutions matter: Professional culture and the development of biogas technology. In: Environmental Innovation and Societal Transitions 8, 20-41.

Zoellner, J.; Schweizer-Ries, P.; Wemheuer, C. (2008): Public acceptance of renewable energies: Results from case studies in Germany. In: Energy Policy 36, 11, 4136-4141. 